\title{
Les Collocutions (ca 1543-1546) de Jan Berthout : variations et répétitions dans un manuel de langue
} Jan Berthout's Collocutions (ca 1543-1546): variations and repetitions in a language manual

Elizaveta Zimont

\section{OpenEdition}

\section{Journals}

Édition électronique

URL : https://journals.openedition.org/dhfles/6659

DOI : $10.4000 /$ dhfles.6659

ISSN : 2221-4038

Éditeur

Société Internationale pour l'Histoire du Français Langue Étrangère ou Seconde

Édition imprimée

Date de publication : 1 décembre 2019

Pagination : 115-140

ISBN : 0992-7654

ISSN : 0992-7654

\section{Référence électronique}

Elizaveta Zimont, «Les Collocutions (ca 1543-1546) de Jan Berthout : variations et répétitions dans un manuel de langue », Documents pour l'histoire du français langue étrangère ou seconde [En ligne], 62-63 | 2019, mis en ligne le 12 avril 2020, consulté le 29 mars 2023. URL : http://journals.openedition.org/ dhfles/6659; DOI : https://doi.org/10.4000/dhfles.6659

Ce document a été généré automatiquement le 29 mars 2023

Tous droits réservés 


\title{
Les Collocutions (ca 1543-1546) de Jan Berthout : variations et répétitions dans un manuel de langue
}

\author{
Jan Berthout's Collocutions (ca 1543-1546): variations and repetitions in a
}

language manual

Elizaveta Zimont

\section{Introduction}

Dans les Pays-Bas méridionaux, les premiers ouvrages destinés à l'enseignement du français langue étrangère datent des XIV et $\mathrm{XV}^{\mathrm{e}}$ siècles (Riemens 1919: 9) ${ }^{1}$. Ce n'est toutefois qu'après l'arrivée de l'imprimerie que la production didactique connaît un véritable essor qui s'inscrit dans un mouvement plus général de description des langues vernaculaires, caractéristique de l'Europe du XVIe siècle (Kaltz 1995: 79; Swiggers 2013 : 50). Parmi les principaux genres didactiques de cette époque (grammaires, recueils de conjugaison, dictionnaires, dialogues-modèles, recueils de lettres-modèles, recueils de proverbes et sentences), les colloques occupent une place à part à cause de leur popularité remarquable qui s'explique en partie par l'importance que la pédagogie humaniste accorde à la conversation (Massebieau 1968: 44-45; Kaltz 1995 : 94-96). Également connus sous le nom de manières de langage ou de manuels de conversation (Kaltz 1992: 124), les colloques présentent un intérêt particulier pour l'histoire de la genèse de l'exercice. À la différence des autres genres didactiques, qui sont conçus essentiellement à des fins de consultation et de mémorisation, les textes dialogués qui forment le noyau des colloques, présupposent l'existence d'une activité pédagogique plus élaborée.

2 Dans le domaine de l'enseignement du français aux néerlandophones, qui se situe au croisement de nouvelles idées humanistes et des pratiques héritées du Moyen Âge tardif, l'exemple le plus célèbre de ces colloques au XVI ${ }^{\mathrm{e}}$ siècle est sans aucun doute le Vocabulaire $\left({ }^{1} 1527\right)$ du maître de langues anversois Noël de Berlaimont $\left({ }^{\circ} \text { ? } † 1531\right)^{2}$. Ses 
dialogues flamands-français inaugurent toute une série d'ouvrages semblables ${ }^{3}$, parmi lesquels les Collocutions ou Tsamencoutinghen (ca 1543-1546) de Jan Berthout n'ont pas encore reçu l'attention qu'elles méritent. Le texte de Berthout, peu connu, présente un intérêt indéniable aussi bien pour l'histoire de l'enseignement du français langue étrangère que pour celle des dialogues didactiques ${ }^{4}$.

3 Après une présentation générale des Collocutions qui s'appuie sur les travaux de De Neve (1962), Claes (2000) et Vanhulst (2005), nous concentrerons notre attention sur les dialogues de Berthout et leur structure. Nous évaluerons ensuite l'importance des Collocutions pour l'histoire de l'enseignement du français langue étrangère afin de mesurer l'influence que cet ouvrage a pu exercer sur d'autres colloques du XVI ${ }^{\mathrm{e}}$ siècle.

\section{Jan Berthout et ses Collocutions}

\section{L'auteur}

La biographie de Jan ou Jean Berthout, dont nous ne connaissons qu'un seul ouvrage, est pauvrement documentée. Quelques renseignements sont éparpillés dans le texte même des Collocutions, pour autant que ces éléments biographiques ne relèvent pas de la fiction littéraire. Fils de Peter ou Pierre Berthout, "clerc suivant la Court» demeurant à Malines, Jan Berthout, "bourgeois natif de la ville de Bruxelles", y fut « maistre d'ecole » vers le milieu du XVI siècle (Berthout 1575 : fol. $1 \mathrm{r}^{\circ}, 33 \mathrm{v}^{\circ}-34 \mathrm{r}^{\circ}, 38 \mathrm{v}^{\circ}$; Vanhulst 2005 : 96). Son école, ouverte aussi bien aux garçons qu'aux filles, se situait « à la porte des Flesches, aupres du pont au miroir ", adresse qui correspond à une impasse aujourd'hui disparue, qui se trouvait à proximité de l'île Saint-Géry à Bruxelles (Berthout 1575 : fol. $14 \mathrm{v}^{\circ}$; Van Wijnendaele 2000 : 5-6). L'édition des Collocutions parue en 1619 renseigne l'auteur comme "feu Jean Berthout». Il s'ensuit que Berthout disparut au plus tard la même année, même s'il est fort probable que notre auteur était décédé bien avant cette date.

\section{Les Collocutions de 1575}

Les Collocutions bien familieres ${ }^{5}$ de 1575, imprimées à Anvers chez Jan (I) van Waesberghe (ca 1528-1590) ${ }^{6}$, présentent une macro-organisation en deux parties ou livres, dont le premier contient des dialogues et des modèles de lettres répartis en quatre chapitres. Les Chapitres I à III comportent trois dialogues : un banquet à la mauvaise saison, un autre banquet en été et un dialogue "pour apprendre à marchander, acheter et vendre ». Le troisième chapitre est complété par un mini-glossaire sur l'expression du nombre, l'expression du temps et une liste des monnaies et devises les plus courantes. Le Chapitre IV réunit des modèles de lettres privées et de pièces juridiques telles qu'un contrat de location, une reconnaissance de dette, un acte d'achat d'une maison, etc. Ce chapitre se termine par une série de formules d'appel pour commencer une lettre, classées selon le rang social du destinataire.

6 Le second livre, intitulé Vocabulaire et doté d'une page de titre séparée, contient un répertoire lexicographique subdivisé en 27 chapitres organisés selon le principe thématique. Le Chapitre XIII «des hommes de mestiers et de leurs instrumentz, ensemble des bruvages et de toutes choses mangeables » est de loin le plus fourni. Ce répertoire est suivi d'une série de prières, des douze articles de la foi, des dix 
commandements et d'une liste de 18 règles pour "apprendre à lire et parler " en français ${ }^{7}$. Hormis ces règles de prononciation et la table des matières qui clôture l'ouvrage, l'ensemble du texte est bilingue, le néerlandais, dans la colonne de gauche, étant placé en regard du français situé, lui, dans la colonne de droite.

\section{Les sources}

7 La macro-organisation des Collocutions rappelle immédiatement celle du Vocabulaire de Noël de Berlaimont qui est également divisé en deux livres : le premier comporte trois dialogues et un chapitre avec des modèles de lettres et d'actes sous forme de "chirographes ${ }^{8}$; le second, un répertoire lexicographique et des règles de prononciation (De Neve 1962 : 127). À partir de 1536, le second livre du Vocabulaire de Berlaimont est complété par des prières, les douze articles de la foi et les dix commandements.

8 Les similitudes concernent non seulement l'organisation globale des deux ouvrages, mais aussi le choix des thématiques explorées dans le premier livre des Collocutions. Les deux premiers dialogues de Berthout mettent en scène des banquets festifs, faisant ainsi écho au premier dialogue de Berlaimont. Le troisième dialogue de Berthout décrit plusieurs scènes sur le marché, sujet traité par Berlaimont dans le deuxième dialogue du Vocabulaire. En ce qui concerne le troisième dialogue de Berlaimont où un personnage vient réclamer à un débiteur le paiement de la dette, la même situation fait l'objet d'une des lettres du Chapitre IV des Collocutions de Berthout. Plus généralement, les modèles de lettres et de "chirographes" du Chapitre IV des deux ouvrages présentent de nombreux parallèles.

Des ressemblances peuvent être constatées aussi bien au niveau de la macroorganisation que dans la trame des dialogues. Voici, à titre d'exemple, un épisode tiré du premier dialogue de Berlaimont. Lors d'un long repas festif, nous voyons un personnage appelé Peeter qui boit à la santé de Tanneken. Par politesse, Tanneken promet d'abord de boire à la santé de Peeter, mais elle s'aperçoit ensuite que Peeter n'a pas entièrement bu son verre, ce qu'elle interprète comme un manque de respect. Voyant que son interlocutrice est vexée, Peeter explique que sa coupe est plus grande que celle de Tanneken et que donc il a bu exactement la même quantité d'alcool qu'elle. Pour désamorcer le conflit, les personnages décident d'échanger leurs récipients.

P. [...] Or cha, je boy une fois à vous et je vous prye pour toute la compaignie et premier pour vostre prochain voisin me ferez vous raison?

T. Ouy, de bon cueur, s'il plaist à Dieu. Or cha, buvez: vous ne l'avez point beu dehors, je le vous verseray encoere une fois plain.

P. Pour quoy feriez vous cela? Ne l'ay je point beu dehors? Combien s'en fault il ? Je le buveray dehors. Regardez là, maintenant est il dehors, maintenant faictes moy raison : vous ne cherchez aultre chose que me tromper.

T. Je ne pouroye boire cecy dehors, j'en ay beaucop trop.

P. Que vous fauldroit il ? Je l'ay bien beu dehors.

T. Vous n'en aviez point aultant que moy, vostre goblet n'estoit point plain.

$[\ldots .$.

P. Il est vray, mais mon goblet est beaucop plus grand que le vostre.

T. Bien, laissez nous changer.

P. J'en suis content, donnez moy vostre goblet ${ }^{9}$.

(Berlaimont 1527 : fol. $\mathrm{C}^{\circ} \mathrm{v}^{\circ}-2 \mathrm{r}^{\circ}$ )

Dans le premier dialogue de Berthout, la même scénette se joue entre Sanson et Ysaias : 
Y. Je boy une fois à vous Sanson.

S. Beuvez tout, je vous feray raison : je vuyderay ce goblet cy.

Y. Non, non : vous vuyderez cette tasse.

S. N'est ce point tout un?

Y. Non dea il y-a grande difference entre vostre goblet \& ceste tasse.

S. Bien, voulons nous changer.

Y. Ouy, changeons.

S. Bien, ce m'est tout un [...].

(Berthout 1575 : fol. $13 \mathrm{v}^{\circ}-14 \mathrm{r}^{\circ}$ )

11 Si la version de Berthout est plus concise que celle de Berlaimont, la trame de l'épisode est identique chez les deux auteurs. Les similitudes entre les Collocutions et le Vocabulaire vont ainsi bien au-delà d'une simple ressemblance typologique, car elles concernent aussi bien la macro-organisation que le contenu concret des dialogues et des modèles de lettres et de documents. La présence d'épisodes très similaires dans les dialogues des deux ouvrages suggère que le Vocabulaire de Berlaimont a pu exercer une influence directe sur les Collocutions de Berthout. Il faut supposer que Berthout, dont les dialogues sont plus longs et plus élaborés, s'inspire du Vocabulaire pour en donner une version perfectionnée.

\section{La datation de l'editio princeps et les éditions}

12 Le manque d'informations au sujet de Berthout rend malaisée la datation des Collocutions dont l'editio princeps n'a pas été conservée. Une datation approximative reste néanmoins possible sur base des références aux évènements et personnages historiques que l'on relève dans l'ouvrage de Berthout, dont les principales sont :

13 - le décès du compositeur Lupus Hellinck ${ }^{10}$ en 1541 mentionné dans le premier dialogue (Berthout 1575 : fol. $4 v^{\circ}$ );

14 - l'incendie qui eut lieu à Anvers en 1541 et qui détruisit l'hôtel de ville, fait divers mentionné dans le troisième dialogue ${ }^{11}$ (Berthout 1575 : fol. 25r ${ }^{\circ}$ );

15 - l'expédition militaire de Maarten van Rossem et de ses troupes qui saccagèrent le Brabant durant l'été $1542^{12}$; un récit précis de ces évènements se trouve dans une lettre du Chapitre IV des Collocutions (Berthout 1575 : fol. 35);

16 - l'annonce du mariage entre le comte Pierre-Ernest Mansfelt et Marguerite de Bréderode, qui eut lieu en $1546^{13}$ (Berthout 1575 : fol. 30º).

17 Comme argument supplémentaire, il faut signaler que tous les modèles rassemblés dans le Chapitre IV des Collocutions sont datés de 1542. En s'appuyant sur ces références historiques, De Neve, puis Vanhulst situent la rédaction des Collocutions dans les années 1540, plus exactement entre 1541 et 1549 au plus tard (De Neve 1962: 131-140; Vanhulst 2005 : 96-97).

18 Cependant, l'édition la plus ancienne conservée date de 1575 ; sa page de titre porte la mention " revue et corrigée ", laissant supposer l'existence d'une édition antérieure à cette date. L'ouvrage figure en effet dans deux inventaires de livres établis en 1569 à l'intention du Conseil des troubles ${ }^{14}$. Le premier, dressé le 16 mars 1569 lors d'une perquisition chez l'imprimeur-libraire bruxellois Michiel van Hamont ${ }^{15}$, mentionne les «Tsamen coutinghen » de «Jan Berthout $»^{16}$. Dans le second, transmis par l'imprimeur Jan (I) van Waesberghe aux échevins de la ville d'Anvers plus tard dans la même année, on retrouve les «Colloques de M. Jan Berthout, en francoys et flamen » classés parmi 
les «Livres en Francoys et en Flamen ensemble pour apprendre et l'un et l'autre langage » (Ledeboer $1869:$ 40-41, 263).

À l'édition non retrouvée d'avant mars 1569, il faut ajouter quatorze autres éditions des Collocutions parues entre le milieu du XVI ${ }^{e}$ et le début du XVIII ${ }^{e}$ siècle, ce qui donne la mesure de la popularité de cet ouvrage ${ }^{17}$. La possibilité de l'existence d'une seizième édition, imprimée à Londres, sera discutée ci-dessous. Au cours de sa longue histoire qui s'étale sur plus d'un siècle et demi, l'ouvrage de Berthout subit plusieurs modifications que l'on peut synthétiser en dégageant trois branches.

La première branche est représentée par l'édition de 1575 qui se différencie par la présence de prières et de règles de prononciation à la fin du second livre. Le répertoire lexicographique de cette édition contient un nombre d'entrées considérablement plus élevé que dans d'autres branches, et l'ordre d'apparition de ces entrées présente de légères déviations par rapport aux autres éditions.

21 La deuxième branche est formée par l'édition de 1699 où l'ordre des livres est inversé. Le répertoire lexicographique, dont le classement a subi une réorganisation profonde, $\mathrm{y}$ apparaît en premier, suivi d'une série de phrases isolées et de formules utiles pour la rédaction d'une lettre. L'ouvrage se termine par quelques modèles de lettres et des formules de politesse identiques à ceux du Chapitre IV des autres éditions. Par contre, les dialogues (Chapitres I à III) y ont été supprimés.

Toutes les autres éditions appartiennent à la troisième branche qui se caractérise par l'absence de prières et de règles de lecture et une nomenclature plus réduite du répertoire lexicographique que dans l'édition de 1575. Deux éditions du XVIII siècle appartenant à cette branche (vers 1704 et 1727) sont privées de répertoire lexicographique.

23 Avec ces quinze ou seize éditions, les Collocutions ne peuvent, certes, pas rivaliser avec le Vocabulaire de Noël de Berlaimont, qui connut plus de 150 rééditions et adaptations. La popularité des Collocutions est toutefois comparable à celle du French School-Master de Claude de Sainliens ${ }^{18}$ (ca 1534-1594), ouvrage anglais-français appartenant au même genre didactique, dont on connait vingt-et-une éditions distinctes (Berec 2013 : 103). Le rayonnement géographique des Collocutions est également plus limité que celui du Vocabulaire de Berlaimont : l'écrasante majorité des éditions conservées (onze) furent imprimées à Anvers; deux proviennent de Dunkerque, une de Louvain. Les lieux d'impression permettent de cerner indirectement l'espace géographique où l'ouvrage de Berthout fut utilisé. Cette aire de circulation relativement réduite s'explique entre autres par le fait que les Collocutions n'ont pas connu d'adaptations multilingues.

\section{Le public-cible des Collocutions}

Dans l'édition de 1575, l'imprimeur van Waesberghe adresse aux lecteurs un avis où il indique que l'ouvrage est «faict à l'utilité de plusieurs, \& principalement de la jeuneße ». Pourtant, les dialogues mettent en scène un monde de personnes adultes, pour la plupart appartenant à la classe marchande, avec les sujets et les préoccupations qui leur sont propres. Les quelques enfants qui y apparaissent ne reçoivent que peu de répliques, autre trait que les Collocutions partagent avec le Vocabulaire de Berlaimont. Grâce aux documents d'archives, nous savons que les Collocutions furent effectivement utilisées dans des établissements scolaires qui offraient une instruction de base comprenant les rudiments de la langue française (Put 1990 : 217-218). Les Collocutions 
figurent ainsi dans un placard intitulé «Prix des livres scolaires courants ${ }^{19}$ » qui fut établi par le Magistrat d'Anvers en vue de fixer les prix des livres d'école les plus usités : «Groote Vocabulaers van Berthout ${ }^{20}, 11$ ou 13 fe., 4 1/2 st. » (Van Heurck 1927 : 133).

\section{La structure des dialogues dans les Collocutions de 1575}

En l'absence de paratextes portant explicitement sur l'utilisation didactique des dialogues de Berthout, nous ne pouvons que construire des hypothèses fondées sur les éléments qui se dégagent de l'analyse d'autres ouvrages similaires. D'une part, la forme du dialogue se prête a priori relativement bien à une mise en scène. Il est néanmoins plus vraisemblable que ces textes étaient destinés à être lus, dictés et mémorisés (Hendrickx 1961: 230). Comme le souligne Merrilees, la forme dialoguée d'un texte didactique ne correspond pas nécessairement à une activité d'apprentissage en forme de dialogue (Merrilees 1985 : 109). D'autre part, la présentation bilingue des dialogues fait penser que la traduction jouait en rôle important dans l'enseignement du français (Hendrickx 1953 : 32). Ainsi, dans ses Colloques ou nouvelle invention de propos familiers (Anvers, 1557), Gabriel Meurier laisse une partie du texte français sans traduction en néerlandais pour offrir au lecteur l'occasion d'apprendre par lui-même « à nager en la traduction des motz François en Flamen» (cité dans De Vreese 1897 : 755). Enfin, la note que Berlaimont insère avant le répertoire lexicographique de son Vocabulaire (1527) suggère que les textes de la première partie de l'ouvrage devaient servir d'exemple pour construire d'autres dialogues sur le même modèle ${ }^{21}$ (Berlaimont 1527 : fol. Giir ${ }^{\circ}$.

En accord avec l'orientation pratique de l'ouvrage, les trois dialogues de Berthout se construisent autour d'une série de situations usuelles telles qu'une messe, un repas de fête (Chapitre I), une promenade à la campagne suivie d'un banquet (Chapitre II), un voyage à Anvers, un séjour dans une auberge et une visite au marché (Chapitre III). Ces situations visent à fournir un cadre adéquat pour présenter le vocabulaire quotidien inséré dans des conversations qui se caractérisent par un enchaînement de formules de politesse et de lieux communs. Les quelques références à des évènements ou des personnages historiques ne sont au fond pas indispensables au déroulement de ces dialogues. Ce trait fut de bonne heure remarqué par les éditeurs de l'ouvrage qui en profitèrent pour mettre les Collocutions «à jour » en remplaçant des personnages et des dates du XVI ${ }^{e}$ siècle par ceux du XVII ${ }^{e}$ siècle, sans que cela eût le moindre impact sur la structure des dialogues (De Neve 1962 : 136 ; Vanhulst 2005 : 106).

\section{Bipartition}

Les trois dialogues de Berthout sont de longueur inégale : alors que le premier s'étale sur vingt-six colonnes et demie, le deuxième et le troisième n'en occupent que quatorze et demie et seize respectivement. La longueur des dialogues peut être mise en corrélation avec le nombre de personnages : le premier dialogue met en scène vingt personnages tandis que dans le deuxième leur nombre est limité à dix et, dans le troisième, à douze. Chaque dialogue s'articule autour de deux parties dont la première est plus courte que la seconde. Dans la première partie, les échanges, impliquant un 
nombre réduit de personnages ${ }^{22}$, s'effectuent entre hommes adultes appartenant, pour l'écrasante majorité, à la même couche de société. La conversation se déroule ainsi dans un registre semi-formel. Par contre, dans la seconde partie des dialogues, où le nombre de personnages atteint son maximum, les profils se diversifient avec l'introduction de catégories d'âge différentes (enfants), de l'autre sexe (femmes) et d'autres couches sociales (domestiques). Cette diversité crée la possibilité de représenter des situations de communication qui sont plus variées sur le plan diaphasique que celles de la première partie.

Le premier dialogue fournit une parfaite illustration de cette bipartition. Dans la première scène, deux connaissances, Hercules et Sanson, se croisent dans une rue de Bruxelles. C'est le Jour de l'an et les deux personnages s'échangent leurs doléances sur la mauvaise nuit qu'ils viennent de passer : le bruit de la musique et des festivités les a empêchés de dormir. Vers neuf heures, les deux amis veulent se rendre à l'église pour y assister à la messe. Ils ont le choix entre «l'Église Sainte-Goule» ou « Nostre Dame de Sablon » et décident finalement d'aller à Notre-Dame du Sablon car ils ont entendu dire que l'Empereur y serait présent. Sur leur chemin, Hercules et Sanson aperçoivent quelques personnalités : le Cardinal de Liège, l'évêque de Tournai, le duc de Lorraine, le Prince d'Orange et quelques autres. Près de l'église, ils sont rejoints par d'autres connaissances, Zacharias et Laurent. À quatre, le groupe entre dans Notre-Dame du Sablon où la messe a déjà commencé. La conversation se poursuit autour des œuvres musicales qu'on est en train d'écouter : les personnages échangent leurs avis sur la beauté des voix et tentent d'identifier les morceaux interprétés en citant des noms de compositeurs célèbres de l'époque. À la sortie de l'église, tous les personnages sont invités au déjeuner chez maître Jacques de la Vallée dont ils croisent par hasard le page. Le groupe s'informe auprès du page sur le nombre d'invités, puis se dirige vers la maison de maître Jacques.

La seconde partie du dialogue met en scène un convivium qui présente plus d'une ressemblance avec le premier dialogue du Vocabulaire de Berlaimont. Les invités arrivent devant la porte de la maison, mais celle-ci est fermée. Lorsqu'ils frappent, on met du temps à leur ouvrir car la servante ne trouve pas les clés. Après les salutations et quelques mots de bienvenue de la part de maître Jacques, l'assemblée commence à s'installer à table. Mais réunir tout le monde n'est pas chose facile: les femmes occupées à la cuisine tardent à rejoindre les hommes pendant que les enfants continuent à jouer dans la cour. Une fois tout le monde rassemblé, commence un festin pantagruélique. Lorsque le vin est épuisé, on envoie le page en chercher en ville. En attendant, les invités proposent de chanter une chanson pour se distraire, sur quoi un deuxième groupe d'invités arrive. Le repas se poursuit jusqu'au dessert après lequel les enfants sont rappelés à table pour dire les Grâces. Les invités félicitent les enfants de leurs talents et c'est à ce moment-là qu'on apprend que la fille de maitre Jacques, la petite Cécile, fréquente l'école à la porte des Flèches, auprès du Pont au miroir. Enfin, le temps de se séparer est venu. Après un échange de remerciements, les invités et les hôtes se disent au revoir.

Cette bipartition du dialogue doit être mise en rapport avec la fréquence variable de certaines formes linguistiques. C'est ainsi que dans la première partie du dialogue, on relève en moyenne une forme d'impératif par page, les formes de la première personne du pluriel étant majoritaires (allons, écoutons). En revanche, dans la seconde partie, où les relations entre les personnages sont davantage hiérarchisées, le nombre 
d'impératifs atteint une moyenne de neuf par page, les impératifs de la première personne étant cette fois-ci minoritaires.

\section{Intégration du lexique thématique}

31 Quels objectifs d'apprentissage se cachent derrière le récit d'une journée festive ? La réponse à cette question n'est pas aisée, dans la mesure où l'auteur n'a pas jugé utile d'accompagner son ouvrage d'une note explicative. Dans la littérature secondaire sur les manières de langage et ouvrages apparentés, les dialogues sont souvent qualifiés d'outils lexicaux. En procurant à l'apprenant un contexte, une situation concrète, les dialogues permettraient de mieux appréhender le lexique thématique (Kibbee 2000 : 190 ; Swiggers $2013: 53)$. Ce lexique thématique, notamment des lexèmes répertoriés dans la partie lexicographique des Collocutions, est effectivement intégré dans les dialogues. Cependant, la principale technique pour l'intégrer consiste à énumérer des notions appartenant à la même catégorie conceptuelle ${ }^{23}$. Dans le premier exemple cidessous, on relève une énumération de couleurs suivie d'une liste de sortes de drap selon leur provenance :

M. Dame n'avez point de bon drap?

A. Ouy, j'en ay de toute sorte, de quelle couleur le vous plait il avoir?

M. Monstrez nous du bon noir, \& aussy du jaune \& du blanc.

A. Regardez ce qui vous duit: voi-là de bon drap de Bruges, \& voi-là drap de Malines, voi-là aussy du drap d'Ypre, ensemble du drap de Bois-le-Duc, aussy de Lile, \& de tous quartiers.

(Berthout 1575 : fol. 27)

La même technique sert à introduire dans le dialogue pas moins de sept sortes de fromage. Quatre d'entre elles sont reprises dans le répertoire lexicographique des Collocutions :

B. Paul, mettez le Permesan au milieu, \& le beurre aux deux costez, tenez là

Estienne, voi-là le fromage d'Angleterre, \& le fromage de Nivelle [...].

A. Ma Damoiselle, c'est un bon formage quel formage est-ce?

D.C. C'est formage de Bethunes, \& cela est fourmage de Lile.

R. Damoiselle, vous avez icy plusieurs sortes de formage.

M. Laurens, voi-là un bon formage de brebis, \& ce formage de Hollande n'est pas

aussy mauvais.

(Berthout 1575 : fol. 13)

Il n'est pas certain que ces « listes » contribuent véritablement à préciser ou à mieux appréhender le sens des termes qui en font partie. Ces énumérations répondent plutôt à une forme de défi dont l'objectif est de citer le plus grand nombre possible de cohyponymes se rapportant à un même terme générique. Par ailleurs, les termes de l'énumération ne figurent généralement qu'une seule fois dans le texte. Leur mémorisation n'est donc certainement pas facilitée par la fréquence de leur emploi dans les dialogues. À cet égard, Berthout se place dans la continuité des manières de langages du Moyen Âge où il n'est pas rare qu'un dialogue soit littéralement entrecoupé par une liste thématique à la façon des nominalia : parties du corps, noms de vêtements, etc. (Merrilees 1985 : 110). Le processus de la rédaction d'un épisode contenant une énumération de co-hyponymes semble avoir pour point de départ la mise en dialogue d'une liste de vocabulaire thématique. 


\section{Répétitions et variations}

34 Si le lexique thématique ne connaît qu'une seule occurrence par lexème, en revanche, beaucoup d'autres composantes des dialogues font l'objet de répétitions. Examinons quelques épisodes récurrents dans le scénario des dialogues. Dans le Chapitre I, deux groupes d'invités s'informent à deux moments différents sur le nombre de personnes assises à table :

S. Monsieur est il assis à table?

G. Non, point encore, mais il y ira incontinent asseoir.

H. Y a il beaucop de gens?

G. Il y en a neuf ou dix.

(Berthout 1575 : fol. 5)

I. [...] Monsieur a il longuement esté a Table?

G. Point fort longuement Jean, on ne fait que mettre le rosty sur la table.

D. $Y$ a il grande compaignie?

G. Ils sont bien quinze ou seize.

(Berthout 1575 : fol. 9r ${ }^{\circ}$ )

Plus tard, chacun des deux groupes est amené à définir l'ordre de leur entrée dans la maison:

P. Entrez Messieurs.

Z. Allez devant Sanson.

S. Allez vous mesme devant Zacharias, car vous y estes mieux cogneu que nous ne sommes.

L. N'est-ce pas tout un ? J'iray moy mesme devant, suivez moy.

(Berthout 1575 : fol. $5 \mathrm{v}^{\circ}-6 \mathrm{r}^{\circ}$ )

Y. Nicolas, entrez.

N. Moy? Je n'y ay nulle connoissance : voicy la premiere fois que j'ay esté en ceste maison.

Y. Allez donc devant Rembert.

R. Bien, je vay devant, suivez moy.

(Berthout 1575 : fol. $9 r^{\circ}$ )

Un autre exemple de répétition dans le scénario est lié à la musique. Aussi bien dans l'église Notre-Dame que chez maître Jacques de la Vallée, les personnages s'informent sur l'identité du compositeur :

Z. C'est un bon motet, qui le peut avoir fait?

L. Lupus, le maitre des chantres de saint Donat à Bruges.

(Berthout 1575 : fol. $4 \mathrm{v}^{\circ}$ )

M.I. Certes voi-là une bonne chanson : qui l'a faite?

R. Je croy que Gombert l'a faite.

M.I. Qui est cestuy-là?

R. C'est le maistre des chantres de l'Empereur.

(Berthout 1575 : fol. $11 v^{\circ}$ )

37 Dans le deuxième dialogue, qui se construit autour de thématiques liées au monde agricole, aux travaux champêtres et à la vie à la campagne, certains passages se font également écho. Dans l'extrait suivant, on parle des dégâts causés par une tempête :

L. Mon oncle a aussy eu fort grand dommage : car il y a bien en son jardin neuf ou dix arbres rompus du grand vent qu'il faisoyt là : il a aussy perdu deux chevaux, \& une vache, qui estoyent au champ [...].

(Berthout 1575 : fol. $17 \mathrm{r}^{\circ}$ )

La même information est ensuite communiquée par un autre personnage, l'oncle évoqué dans le passage ci-dessus : 
D. Ouy vrayement ma Damoiselle, il y a en mon jardin trois cerisiers rompus, deux pommiers, un prunier, \& quatre poyriers : j'ai aussy perdu aucunes de mes bestes : à savoir deux chevaux, \& une vache.

(Berthout 1575 : fol. 20vº répétitions intentionnelles sont révélatrices de ce que l'auteur pouvait concevoir comme objectif d'apprentissage. Le scénario des dialogues est conçu de manière que les épisodes puissent être réitérés avec des modifications légères. Notons que si une situation se répète, elle est à chaque fois jouée par des personnages différents. Comme le montrent les exemples cités ci-dessus, les répétitions fournissent la possibilité de formuler le même contenu sémantique, et parfois pragmatique, en recourant à des moyens d'expression linguistiques variés, tant sur le plan du vocabulaire que sur le plan des constructions syntaxiques. Le dialogue se compose ainsi d'une série de courts blocs de répliques qui se répètent deux ou trois fois à l'intérieur d'un chapitre, voire parfois entre chapitres. Chaque bloc correspond à une tâche communicative bien spécifique comme, par exemple, saluer les invités, faire un compliment, etc. En faisant abstraction du scénario du premier dialogue, nous pouvons dégager plusieurs types de tâches communicatives récurrentes dont voici les plus fréquentes :

À titre d'exemple, le premier dialogue contient onze épisodes où un personnage demande à un autre de transmettre un message ou une injonction. Ces interactions se déroulent entre personnages qui appartiennent à des catégories différentes : entre le maitre et le serviteur, entre le serviteur et les enfants, etc. Les répliques du messager sont particulièrement intéressantes car les structures qu'il utilise varient en fonction du destinataire. Comparez la première scène où un domestique transmet le message de son maître à la femme d'un des invités avec la seconde où un autre domestique transmet un message au jeune fils du maître :

M.I. [...] Allez vous en parler à la femme de Laurens, \& dites luy qu'elle vienne disner avec moy.

G. Bien Monsieur. [...] Mon Seigneur vous prie, de vouloir venir disner avec luy.

(Berthout 1575 : fol. $6 \mathrm{r}^{\circ}$ )

M.I. [...] Guillot, dites à Thomas qu'il vienne dire le Benedicite.

G. Bien Monsieur. Thomas, ou estes vous?

T. Que dites vous?

G. Venez dire le Benedicite.

(Berthout 1575 : fol. $6 \mathrm{v}^{\circ}-7 \mathrm{r}^{\circ}$ )

La réitération des tâches communicatives met en évidence les paramètres linguistiques qui varient en fonction du locuteur et du destinataire. Cet aspect des dialogues vise à favoriser l'acquisition de la variation diaphasique dans la langue-cible de l'apprenant. 


\section{Les Collocutions comme source d'inspiration}

\section{Collocutions n'ont pas connu d'adaptations multilingues, cela n'empêche que l'ouvrage} s'est fait une place sur la scène internationale par un détour qui nous mène vers l'Angleterre élisabéthaine. Dans la seconde moitié du XVI' siècle, le marché anglais des manuels pour apprendre le français était dominé par les ouvrages de Claude de Sainliens dont nous avons déjà évoqué le French School-Master. Cet ouvrage, dont la première édition parue avant 1566 est aujourd'hui introuvable, renferme parmi d'autres composantes deux dialogues qui ont souvent été prisés pour leur authenticité et pour leur vivacité (Farrer 1908 : 21, 26 ; Berec 2013 : 104). Voici un extrait du résumé que Farrer donne du premier dialogue du French School-Master:

On se lève tôt le matin réveillé par les musiciens ambulants. On se promène en écoutant le sermon à la croix de Saint-Paul; on assiste à l'office. Puis on rentre dîner chez un marchand. [...] Les enfants [...] ne manquent pas de parler de leur maître M. Claude de Sain-Liens. Le dîner se prolonge. On chante [...]. (Farrer 1908: 26-27).

Les ressemblances avec le scénario du premier dialogue de Berthout sont pour le moins frappantes. Dans les deux cas, on est réveillé par les musiciens, on se rend à l'église : chez le catholique Berthout, pour y assister à la messe et écouter de la musique ; chez de Sainliens, qui rédigea son texte dans l'Angleterre anglicane, pour écouter le sermon. Le premier dialogue du French School-Master suit ainsi la trame du premier dialogue de Berthout, dont de Sainliens reproduit de nombreux épisodes et même plusieurs répliques concrètes, tout en adaptant leur style et les realia à son public-cible anglais. Nous ne donnerons ici que trois exemples parlants de ces épisodes parallèles :

H. Bon jour, \& bonne santé vous doint Dieu Sanson.

S. Et à vous aussy Hercules, bon jour \& bon nouvel an vous doint Dieu. N'avez vous pas ouï ceste nuit le plaisir des jeux, qu'on faisoit par les ruës?

H. Ouy, n'auroy-je point? Je n'ay de toute la nuit gueres dormy: car depuis les douse heures, je n'ay autre chose ouy que chanter \& joüer, avec plusieurs sortes d'instrumens.

(Berthout 1575 : fol. $2 r^{\circ}$ )

Dieu vous doint bon jour compere.

Et à vous aussi : comment vous portes vous à ce matin ? [...]

Bien, Dieu mercy, sauf que je n'ay peu dormir toute la nuict.

Pourquoy? Qui vous a empesché?

Comment, n'aves vous pas ouï les menestriers \& jouëurs d'instruments, qui joüoyent si melodieusement devant l'hostel de la ville, depuis minuict jusques au point du jour?

(Desainliens 1972 [1573] : 67-69)

H. Dites Sanson, ne savez vous qui c'estoient?

S. Non certes: il me semble que c'estoient Alemans, car ceux de nostre pays ne joüent pas en telle sorte comme ils joüoient : ou il faloit que ce fussent des gens de l'Empereur, selon mon entendement.

(Berthout 1575 : fol. $2 \mathrm{v}^{\circ}$ )

Mais à propos, qui estoient ces chantres et jouëurs d'instruments?

Je ne sçay certes : si d'aventure ce n'estoient les menestriers de la ville, avec ceux de la Rynne, entremeslez de voix d'Italiens \& Anglois, qui chantoient fort harmonieusement.

(Desainliens 1972 [1573] : 69)

H. Ilz chantent bien, je n'ouï onc mïeux chanter. 
Z. Ne moy aussy: je les orroye plus volontiers chanter, que je ne mangeroye ou beuvroie.

L. Et je les orroye plus volontiers chanter, quand j'auroye bien mangé \& bien beu.

(Berthout $1575:$ fol. $4 v^{\circ}$ )

Cela peut estre : car, à dire la verité, je n'ouï jamais mieux chanter.

Oyez, voila un bon verset.

Je vous promes que je les orroye plus volontier chanter, que boyre ou manger.

Je ne suis pas de vostre opinion: car il me semble que je les orroye plus allaigrement, si j'avoye bien disné [...].

(Desainliens 1972 [1573] : 77) entre le French School-Master et les Collocutions. Ajoutons que la subdivision du répertoire lexicographique en chapitres ainsi que l'ordre de classement des entrées sont quasiment identiques dans les deux ouvrages. Par conséquent, si la datation des Collocutions est exacte (ca 1543-1546), il faut supposer que de Sainliens a connu l'ouvrage de Berthout ou un de ses dérivés suffisamment proche de l'original pour que les ressemblances restent évidentes.

Par ailleurs, nous savons que lors de la rédaction du French School-Master, de Sainliens a consulté un ou plusieurs livres conçus pour l'enseignement du français aux étrangers et rédigés dans les Pays-Bas espagnols. Dans l'avis au lecteur qui ouvre le French SchoolMaster, de Sainliens formule une critique acerbe contre un tel ouvrage didactique :

Je ne diray rien d'un nouveau livre venu d'Anvers, \& dernierement imprimé à Londres: acause que ne gardant ryme ne raison soit en son parler, phrase, orthographe, maniere de converser \& communiquer entre gens d'estat: \& ce pendant qu'il pendarise en son jargon, il monstre de quel cru il est sorti [...] Qu'il enseigne donc son beau language aux Flamans, s'accomodant à leur phrase : aux Bourguignons \& Hennuyers [...]. (Desainliens 1972 [1573] : fol. A6-A7).

51 Si de Sainliens omet de mentionner le titre du livre qu'il critique, on retiendra néanmoins de ce passage que des ouvrages didactiques rédigés dans les Pays-Bas méridionaux étaient exportés dans la seconde moitié du XVI siècle vers l'Angleterre et parfois même réimprimés sur place ( $c f$. Lambley $1920: 240-241)^{24}$. Dans la biographie qu'il a consacrée à de Sainliens, Farrer se demande si l'ouvrage critiqué peut être identifié à une édition du Vocabulaire de Berlaimont (Farrer 1908: 27). Or, les fortes ressemblances entre le French School-Master et les Collocutions, que nous venons de mettre en lumière, nous permettent d'avancer l'hypothèse selon laquelle l'ouvrage critiqué par de Sainliens doit être identifié au texte de Berthout. Il faut alors supposer que les Collocutions ont été réimprimées au moins une fois à Londres ${ }^{25}$. Malheureusement, aucune trace de cette édition n'a pu être découverte à ce jour.

$\mathrm{Au}$ XVIIe siècle, le French School-Master fit l'objet d'une adaptation où le texte anglais fut supplanté par une traduction en néerlandais : Vocabulaire François et Flamend, de Maistre Claude Holyband. Tres-profitable \& bien facile pour apprendre la langue Françoise (Rouen, 1647). Les dialogues de Berthout ont ainsi connu une troisième vie.

\section{Conclusion}

Les Collocutions de Jan Berthout, qui s'inspirent de la version initiale du Vocabulaire de Noël de Berlaimont, jouèrent un rôle important dans l'enseignement institutionnel du français langue étrangère durant le XVI ${ }^{e}, \mathrm{XVII}^{e}$ et le premier tiers du XVIII ${ }^{\mathrm{e}}$ siècles dans les Pays-Bas méridionaux. Le succès de l'ouvrage se traduit par le nombre d'éditions

Documents pour l'histoire du français langue étrangère ou seconde, 62-63 | 2019 
conservées, mais aussi par le fait que les Collocutions ont servi, directement ou indirectement, de source à un autre ouvrage didactique de la seconde moitié du XVI ${ }^{\mathrm{e}}$ siècle : le French School-Master de Claude de Sainliens. Ces éléments prouvent que les Collocutions étaient appréciées de leur temps pour le choix des thématiques et la façon dont elles sont traitées. L'analyse des dialogues des Collocutions a révélé que ceuxci se composent d'une série de blocs de répliques dont chacun s'articule autour d'une tâche communicative bien précise, les tâches pouvant être réitérées à plusieurs reprises dans des situations de communication diversifiées. Cet agencement modulaire permet de faire interagir des personnages appartenant à des catégories différentes, ce qui implique le recours à des registres variés. Bien plus qu'un simple support pour la présentation du vocabulaire, le dialogue chez Berthout vise à offrir à l'apprenant un outil qui le guide dans la variation diaphasique et pragmatique de la langue-cible.

\section{BIBLIOGRAPHIE}

\section{Sources primaires}

Bruxelles, Archives générales du Royaume, Conseil des troubles, registre 28.

BERLAIMONT, Noël de (1527). Vocabulaire de nouveau ordonne/ et de rechief recorrige pour aprendre legierement a bien lire escrire/ et parler Fransoys \& flameng/lequel est miz tout la plus part/par personnaiges. Anvers : Jacob van Liesvelt.

BERTHOUT, Jan (1575). Collocutions bien familieres, compozees par Jean Berthout maistre d'ecole à Bruxelles, ausquelles est ajouté un Vocabulaire, divisé par Chapitres : Nouvellement reveu \& corrigé, par E. de W. Anvers : Jan (I) van Waesberghe.

DESAINLIENS, Claude (1972 [1573]). The French School-Master. Menston : The Scolar Press Limited.

\section{Sources secondaires}

ADAM, Renaud (2018). « Le commerce du livre à Bruxelles au XVI ${ }^{\mathrm{e}}$ siècle ». Histoire et civilisation du livre : revue internationale, $14,37-51$.

BEREC, Laurent (2013). «Claude de Sainliens, un huguenot bourbonnais au temps de Shakespeare : un résumé très succinct, quelques ajouts et des pistes de recherche ». Documents pour l'histoire du français langue étrangère et seconde, 50, 101-115.

CLAES, Frans s.j. (2000). « Vocabulaires et livres de conversation pour apprendre le français aux Pays-Bas espagnols entre 1550 et 1700 ». In Jan De Clercq, Nico Lioce \& Pierre Swiggers (dir.). Grammaire et enseignement du français, 1500-1700. Louvain : Peeters, 217-235. COlombo TIMELl, Maria (1998). « Dialogues et phraséologie dans quelques dictionnaires plurilingues du XVI ${ }^{\mathrm{e}}$ siècle (Berlaimont et Solenissimo Vochabulista) ». Documents pour l'histoire du français langue étrangère et seconde, 22, 27-63. 
DE CLERCQ, Jan (1997). « Gabriel Meurier, een XVIe-eeuws pedagoog en grammaticus in Antwerpen ». Meesterwerk, 10, 29-46.

DE NEVE, O. (1962). « Over een ‘Vocabulaer' van de Brusselse schoolmeester Jan Berthout ». De Gulden Passer, 40, 125-144.

DE VREESE, Willem (1897). « Meurier, Gabriel ». Biographie Nationale de Belgique, XIV, col. 700-763.

FARRER, Lucy (1908). Un devancier de Cotgrave. La vie et les œuvres de Claude de Sainliens, alias Claudius Holyband. Paris : Honoré Champion.

GESSLER, Jean (1931). Het Brugsche Livre des Métiers en zijn navolgingen. Vier aloude conversatieboekjes om Fransch te leeren. 6 fasc. Bruges : Sint-Catharina Drukkerij.

HENDRICKX, Antoine (1953). « De stand van de algemene methodiek en didactiek van de Franse taal in de $16^{\mathrm{e}}$ en $17^{\mathrm{e}}$ eeuwen ». Handelingen van het Vlaams filologencongres, XX, 29-34.

HENDRICKX, Antoine (1961). «Franse school- en leerboeken in de $16^{\mathrm{e}}$ en de $17^{\mathrm{e}}$ eeuw ». Paedagogica historica, 1, 225-243.

HENRARD, Paul (1894-1895). « Mansfelt, Pierre-Ernest, comte de ». Biographie Nationale de Belgique, XIII, col. 382-394.

JOBY, Christopher (2015). The Dutch Language in Britain (1550-1702) : A Social History of The Use of Dutch in Early Modern Britain. Leyde/Boston : Brill.

KALTZ, Barbara (1992). «Étude historiographique des Manières de Langage ». In Anders Ahlqvist et al. (dir.). Diversions of Galway. Papers on the History of Linguistics from ICHoLS V, Galway, Ireland, 1-6 September 1990. Amsterdam/Philadelphie : John Benjamins, 123-133.

KALTZ, Barbara (1995). «L'enseignement des langues étrangères au XVI ${ }^{\mathrm{e}}$ siècle : structure globale et typologie des textes destinés à l'apprentissage des vernaculaires ». Beiträge zur Geschichte der Sprachwissenschaft, 5(1), 79-105.

KIBBEE, Douglas (2000). « From Holyband to Mauger : teaching French in 17th-century England ». In Jan De Clercq, Nico Lioce \& Pierre Swiggers (dir.). Grammaire et enseignement du français, 1500-1700. Louvain : Peeters, 179-195.

LAMBLEY, Kathleen (1920). The Teaching and Cultivation of The French Language in England during Tudor and Stuart Times. Manchester : University Press.

LEDEBOER, Adrianus Marinus (1869). Het geslacht van Waesberghe : eene bijdrage tot de geschiedenis der boekdrukkunst en van den boekhandel in Nederland. La Haye/Utrecht : Martinus Nijhoff/J.L. Beijers.

MASSEBIEAU, Louis (1968 [1878]). Les colloques scolaires du seizième siècle et leurs auteurs (1480-1570). Genève : Slatkine Reprints.

MERRILEES, Brian (1985). « Le dialogue dans la méthodologie du français langue seconde au Moyen Âge ». In Pierre L. Léon \& Paul Perron (dir.). Le dialogue. Ottawa : Didier, 106-115.

MINERVA, Nadia, MANDICH, Anna Maria et PELLANDRA, Carla (dir.) (1998). Les dialogues dans les enseignements linguistiques : profil historique. Documents pour l'histoire du français langue étrangère ou seconde, 22. Paris : SIHFLES.

PABLO NÚÑEZ, Luis (2010). El arte de las palabras. Diccionarios e imprenta en el Siglo de Oro. 2 vol. Mérida : Editora Regional de Extremadura.

RIEMENS, Kornelis Jacobus (1919). Esquisse historique de l'enseignement du français en Hollande du XVI au XIXe siècle. Leyde : Société d'éditions A.W. Sijthoff. 
RIEMENS, Kornelis Jacobus (1924). Étude sur le texte français du Livre des Métiers. Supplément à l'Esquisse historique de l'enseignement du Français en Hollande du XVI ${ }^{e}$ au XIX siècle. Paris : L. Arnette. ROOBAERT, Edmond (2009). « Michiel van Hamont. Hellebaerdier van de keizer, rederijker en drukker van de koninklijke ordonnanties en plakkaten ». In Frank Daelemans \& Ann Kelders (dir.). Miscellanea in memoriam Pierre Cockshaw (1938-2008). Bruxelles : Archives et Bibliothèques de Belgique, 465-485.

SWIGGERS, Pierre (2013). «Regards sur l'histoire de l'enseignement du français aux Pays-Bas (XVI - XVIII siècles) ». Documents pour l'histoire du français langue étrangère ou seconde, 50, 49-79. SWIGGERS, Pierre \& ZIMONT Elizaveta (2015). « Dutch-French bilingual lexicography in the Early Modern period. A checklist of sources ». Beiträge zur Geschichte der Sprachwissenschaft 25(1), 110-148.

VAN HEURCK, Émile (1927). Voyage autour de ma Bibliothèque. Anvers : De Cocker.

VAN WIJNENDAELE, Jacques (2000). « Hypothèses sur le premier castrum de Bruxelles ». Cercle d'Histoire de Bruxelles, 17(2), 3-9.

VANDER LINDEN, Herman (1908-1910). « Rossem, Martin van ». Biographie Nationale de Belgique XX, col. 145-159.

VANHULST, Henri (2005). «La musique dans les manuels de conversation bilingues de la Renaissance : Les Seer gemeyne Tsamencoutingen / Collocutions bien familieres de Jean Berthout ». Revue belge de musicologie / Belgisch Tijdschrift voor Muziekwetenschap, 59, 93-124.

VERDEYEN, René (1925-1935). Colloquia et Dictionariolum septem linguarum, gedruckt door Fickaert te Antwerpen in 1616 [réimpression d'Anvers 1616]. 3 vol. Anvers : Uitgave van de Vereeniging der Antwerpsche Bibliophilen.

\section{NOTES}

1. Sur ces ouvrages conçus en forme de dialogues bilingues ou de simples listes de mots français mis en regard de leurs traductions flamandes, $c f$. Riemens (1924), Gessler (1931).

2. Sur l'ouvrage de Berlaimont, dont la version originale comprenait trois dialogues, $c f$. Verdeyen (1925-1935), Pablo Núñez (2010).

3. Cf. Glaude Luython, Dictionaire en Franchois \& Flameng ou bas allemant tresutile pour apprendre les deux langages (Anvers, 1552); Gabriel Meurier, Colloques ou nouvelle invention de propos familiers (Anvers, 1557), Propos pueriles en François et Flamen (Anvers, 1561), La Guirlande des jeunes filles (Anvers, 1564), La Foire des enfants d'Israel, en François et Flamen (Anvers, 1580), Le Perroquet mignon des petits enfants François-Flameng (Anvers, 1580) ; Jacques Grevin, La premiere et la seconde partie des dialogues françois pour les jeunes enfans (Anvers, 1567) ; Gérard de Vivre, Douze dialogues, traitans de diverses manieres, tres-propres aux nouveaux apprentifs de la langue françoise (Anvers, 1574), etc. (Hendrickx 1961 : 228-230 ; De Clercq 1997 : 30-31).

4. Sur l'histoire de l'utilisation des dialogues dans l'enseignement des langues en Europe, $c f$. notamment Documents pour l'histoire du français langue étrangère ou seconde 22 (Minerva, Mandich \& Pellandra [éds] 1998).

5. En néerlandais Seer ghemeyne Tsamencoutinghen.

6. Sur cet imprimeur anversois qui publia plusieurs ouvrages didactiques destinés à l'enseignement du français langue étrangère, $c f$. Ledeboer (1869).

7. Il s'agit essentiellement de règles de correspondance entre les graphies et les sons et de quelques règles morphophonétiques. 
8. C'est-à-dire des modèles de conventions, obligations et quittances qui devaient ensuite être revêtues de signatures autographes des personnes impliquées. Cf. la description du Chapitre IV chez Berthout ci-dessus.

9. Pour faciliter la lecture des extraits, nous avons modernisé l'emploi des lettres $\langle\mathrm{u}\rangle$ et $\langle\mathrm{v}\rangle$, $<\mathrm{i}>$ et $<j>$ ainsi qu'ajouté l'accent grave sur la lettre $<a>$. Les abréviations ont été résolues. Nous avons en outre restitué les majuscules manquantes et introduit des apostrophes et des signes de ponctuation là où cela a paru nécessaire à la compréhension des textes.

10. Lupus (Wulfaert) Hellinck ou Helling (ca 1494-1541) est un compositeur d'école flamande qui fut «maître des chantres » à l'église Saint-Donatien de Bruges (De Neve 1962: 139; Vanhulst $2005: 102-103)$.

11. Cf. De Neve (1962: 133).

12. Van Rossem défendait alors les intérêts des ducs de Gueldre contre les prétentions de Charles Quint. Après la défaite définitive des ducs de Gueldre, Van Rossem passa au service de Charles Quint en 1543 (Vander Linden 1908-1910 : 150-153).

13. Cf. Henrard (1894: 382).

14. Un tribunal d'exception, dirigé par le duc d'Albe, qui fonctionna dans les Pays-Bas espagnols entre 1567 et 1576 et qui ordonna une série de mesures de répression visant entre autres à identifier et répertorier les ouvrages hérétiques alors disponibles sur le marché. Les inventaires de livres établis dans le cadre des perquisitions menées auprès d'imprimeurs et libraires de plusieurs villes constituent une source précieuse pour l'histoire du livre dans les Pays-Bas méridionaux au XVIe siècle (Adam 2018 : 45).

15. Sur Michiel van Hamont (fl. 1554-1585), imprimeur juré du roi, installé à Bruxelles, $c f$. Roobaert (2009).

16. Bruxelles, AGR, Conseil des troubles, 28 , fol. $28 \mathrm{r}^{\circ}$. Nous tenons à remercier M. Renaud Adam de nous avoir signalé cette source.

17. Pour un aperçu incomplet des éditions, cf. Vanhulst (2005) et Swiggers \& Zimont (2015). Comme cela est courant pour les livres scolaires du XVIe et XVIIe siècles, les exemplaires conservés des différentes éditions sont rarissimes.

18. Parfois orthographié Desainliens, alias Claudius Holyband.

19. Taxatie oft prijsen vande ghemeyne School-Boecken.

20. C'est à dire " grands vocabulaires de Berthout ».

21. «Après avoir vu dans le premier livre en guise d'exemple plusieurs manières de parler français présentées en forme de conversations ordinaires, maintenant dans ce second livre vous avez des mots communs rangés par ordre alphabétique comme matière pour faire vous-mêmes d'autres conversations " (notre traduction).

22. Entre deux et six selon les épisodes et les dialogues.

23. Cf. les observations similaires de Colombo Timelli (1998: 39) à propos du Vocabulaire de Berlaimont.

24. Dans la seconde moitié du XVI ${ }^{\mathrm{e}}$ siècle, plusieurs imprimeurs établis à Londres et à Norwich publient des livres en néerlandais ou flamand. Cependant, comme le remarque Joby, il n'est pas toujours aisé de savoir si un ouvrage a été imprimé en Angleterre ou dans les Pays-Bas, car, pour éviter la censure, les imprimeurs utilisent fréquemment des adresses fictives (Joby 2015 : 18, 140-144).

25. Plusieurs témoignages semblent indiquer que les maîtres des écoles néerlandaises («Dutch schools»), qui assuraient l'enseignement du néerlandais aux enfants des communautés néerlandophones d'Angleterre à l'époque élisabéthaine, étaient également chargés d'enseigner le français (Joby $2015: 176-179$; $c$. Lambley 1920 : 239). Dès lors, il est probable que les Collocutions ont été importées pour enseigner le français langue étrangère à des néerlandophones installés à Londres. 


\section{RÉSUMÉS}

Les Collocutions bien familieres (ca 1543-1546) du maitre bruxellois Jan (Jean) Berthout s'inscrivent dans une longue série de colloques bilingues flamands-français produits au XVI ${ }^{\mathrm{e}}$ siècle dans les Pays-Bas méridionaux et destinés à l'enseignement du français langue étrangère. En nous intéressant à cet ouvrage jusqu'ici peu étudié, nous nous centrons sur les trois dialogues de la première partie des Collocutions qui s'inspirent de la version originale du Vocabulaire (1527) de Noël de Berlaimont. S'il s'avère impossible de déterminer avec précision le type d'activité d'apprentissage pour lequel ces dialogues sont conçus, l'analyse de leur structure révèle que, bien plus que de simples outils lexicaux, ces textes favorisent l'acquisition de la variation diaphasique et pragmatique dans la langue-cible. Cet objectif est atteint grâce à une organisation modulaire où les mêmes tâches communicatives sont réitérées dans des contextes de communication variés. La longue histoire éditoriale de l'ouvrage ainsi que l'existence d'une version adaptée en anglais attestent du succès de cette formule modulaire.

The Collocutions bien familieres (ca. 1543-1546) by the Brussels language master Jan (Jean) Berthout are part of a long series of bilingual Flemish-French colloquies produced during the sixteenth century in the Southern Low Countries. This article focuses on the three dialogues from the first part of Collocutions, which draw upon the original version of the Vocabulaire (1527) by Noël de Berlaimont. Although it appears hard to determine the exact nature of learning activities for which the dialogues are intended, the analysis of their structure reveals that, far from being simple lexical tools, these texts foster the acquisition of diaphasic and pragmatic variation in the target language. This goal is achieved by means of a modular text structure where the same communication tasks appear repeatedly in various communicative contexts. The considerable number of editions of the work as well as the existence of an adapted English version reflect the success of its modular design.

\section{INDEX}

Keywords : Berthout, sixteenth century, Southern Low Countries, French as a foreign language, colloquia, dialogues, vocabulary acquisition, stylistic variation

Mots-clés : Berthout, XVIe siècle, Pays-Bas méridionaux, français langue étrangère, colloques, dialogues, acquisition du vocabulaire, variation diaphasique

\section{AUTEUR}

\section{ELIZAVETA ZIMONT}

Université de Liège \& FNRS elizaveta.zimont@uliege.be 